\title{
Canonical and noncanonical equilibrium distribution
}

\author{
Mario Annunziato, ${ }^{1, *}$ Paolo Grigolini, ${ }^{1,2,3, \dagger}$ and Bruce J. West ${ }^{4, \$}$ \\ ${ }^{1}$ Dipartimento di Fisica dell'Università di Pisa and INFM, Piazza Torricelli 2, 56127 Pisa, Italy \\ ${ }^{2}$ Istituto di Biofisica CNR, Area della Ricerca di Pisa, Via Alfieri 1, San Cataldo 56010 Ghezzano-Pisa, Italy \\ ${ }^{3}$ Center for Nonlinear Science, University of North Texas, P.O. Box 305378, Denton, Texas 76203-5378 \\ ${ }^{4}$ U.S. Army Research Office, Research Triangle Park, North Carolina 27709
}

(Received 24 October 2000; published 19 June 2001)

\begin{abstract}
We address the problem of the dynamical foundation of noncanonical equilibrium. We consider, as a source of divergence from ordinary statistical mechanics, the breakdown of the condition of time scale separation between microscopic and macroscopic dynamics. We show that this breakdown has the effect of producing a significant deviation from the canonical prescription. We also show that, while the canonical equilibrium can be reached with no apparent dependence on dynamics, the specific form of noncanonical equilibrium is, in fact, determined by dynamics. We consider the special case where the thermal reservoir driving the system of interest to equilibrium is a generator of intermittent fluctuations. We assess the form of the noncanonical equilibrium reached by the system in this case. Using both theoretical and numerical arguments we demonstrate that Lévy statistics are the best description of the dynamics and that the Lévy distribution is the correct basin of attraction. We also show that the correct path to noncanonical equilibrium by means of strictly thermodynamic arguments has not yet been found, and that further research has to be done to establish a connection between dynamics and thermodynamics.
\end{abstract}

DOI: 10.1103/PhysRevE.64.011107

PACS number(s): 05.20.-y, 05.45.-a, 03.65.Sq

\section{INTRODUCTION}

The problem of the thermodynamic foundation of anomalous diffusion was raised by Montroll and Shlesinger [1], who found that the use of the ordinary method of entropy maximization would yield the process of Lévy diffusion by using an ad hoc, and so unsatisfactory, logarithmic constraint. This conclusion seems to leave open the problem of the thermodynamic foundation of Lévy processes. In fact the issue of the thermodynamic foundation of strange kinetics has been addressed more recently by other authors: this issue became more popular in the last few years as a result of the work of Zaslavsky [2], Zanette and Alemany [3], Tsallis et al. [4] and more recently of Buiatti et al. [5]. The paper of Zaslavsky shows that strange kinetics can be responsible for paradoxical effects. This author shows that two chaotic billiards, coupled to one another through a small hole in the wall separating one billiard from the other, result in so strong a violation of the condition of equal distribution as to suggest the occurrence of a Maxwell's demon effect. Zaslavsky states that these effects, generated by the strange kinetics of weak chaos, oblige us to rethink the foundation of thermodynamics.

The papers of Refs. [3-5] are based on the conjecture that the nonextensive thermodynamics proposed by Tsallis in his 1988 pioneering paper [6] might account for the physical effects generated by strange kinetics. The intriguing issue of whether the nonextensive thermodynamics of Tsallis might also be the proper kind of thermodynamics necessary to explain the Maxwell's demon effect of Zaslavsky was ad-

\footnotetext{
*Email address: annunzia@df.unipi.it

†Email address: grigo@unt.edu

${ }^{\ddagger}$ Email address: WestB@ aro-emh1.army.mil
}

dressed by Aquino et al. [7]. These authors found that the adoption of nonextensive thermodynamics is not incompatible with a slow transition to the final equilibrium condition, but it is not the correct explanation of the Maxwell's demon effect. In fact, this striking effect is proved to be possible as a form of genuine equilibrium, a conclusion confirmed by the more recent work of Ref. [8].

The present paper deals with the issue of equilibrium again, from a perspective different from that of the Maxwell's demon effect of Refs. [2,7,8], though, which is convenient for us to illustrate in detail. According to Tsallis [4] canonical equilibria are singular in a more general form: canonical equilibrium, the distributions that form the basis of equilibrium statistical mechanics, are not generic. Rather, canonical equilibria are singular in a more general form of equilibrium, called a generalized canonical equilibrium $[9,10]$. A number of recent papers elaborate on this idea [1113], but we find [13] to be of special interest addressing as it does the foundations of both canonical and noncanonical equilibria. Rajagopal and Abe [13] prove that the equilibrium described by the canonical distribution is not uniquely determined by the microcanonical distribution, as one finds in text books. In fact if the phase space has a fractal, rather than a smooth structure, a noncanonical distribution will result. However, although canonical equilibria are not unique, and noncanonical equilibria are possible, one might conclude on the basis of the arguments in [13] that the noncanonical distribution is uniquely of the form established by Tsallis and co-workers $[6,9]$. As attractive as the probabilistic and entropic arguments of Ref. [13] are, they are not indisputable and in fact we find herein, using dynamical arguments, that this is not the case.

We show that the adoption of a dynamical approach to thermodynamical equilibrium yields a different conclusion. First of all, we argue that the nonextensive condition based 
on memory, and probably that resting on long-range correlations as well, has the striking effect of making the role of dynamics much more important than in the case of ordinary statistics. The dynamic approach, in these nonextensive conditions, generates a form of noncanonical equilibrium that, however, departs from the generalized canonical form prescribed by nonextensive thermodynamics. To make it easier for the reader to follow our arguments and to understand the purpose of the paper, illustrated in Sec. ID, we shall first discuss the different natures of the entropic, dynamic, and stochastic approaches to equilibrium.

\section{A. Nonextensive entropic indicator}

The argument for the nonextensive thermodynamics of Tsallis goes as follows. First of all, the conventional entropy of Gibbs,

$$
S(\Pi) \equiv-\int d x \Pi(x) \ln \Pi(x),
$$

is replaced by the nonextensive entropic indicator

$$
S_{q}(\Pi) \equiv \int d x \frac{1-\Pi(x)^{q}}{q-1} .
$$

Second, we have to apply a method of entropy maximization under given physical constraints to determine the most plausible shape of the unknown probability density function $\Pi(x)$ [14]. The first constraint is on the normalization of the distribution $\Pi(x)$,

$$
\int d x \Pi(x)=1
$$

The second constraint is on the first moment of the variable $x$ itself. According to the most recent prescription of Ref. [15] the constraints on $x$ must be applied [16] on the mean value $U_{q}$ defined by

$$
U_{q} \equiv \frac{\int d x x \Pi(x)^{q}}{\int d x \Pi(x)^{q}} .
$$

It has to be pointed out that in Ref. [15] the physical meaning of $x$ is that of energy. Here, we shall interpret $x$ as the "coordinate" of an overdamped particle driven by a fluctuationdissipation process resulting from the interaction with a nonconventional "thermal bath." When the friction term can be neglected we are expected to recover the results of the earlier work of Ref. [5]. Thus, for the same reasons [17] as those illustrated in Ref. [5] we set a constraint on the first moment of $|x|$. The result of entropy maximization subject to the imposed constraints of Eq. (4) yields

$$
\Pi(x)=\left[1-\frac{(1-q) \widetilde{b}\left(x-U_{q}\right)}{\int d x \Pi(x)^{q}}\right]^{1 /(1-q)} / Z_{q},
$$

where $Z_{q}$ is a normalization factor and $\beta$ is a constant value stemming from the Lagrange multiplier associated with the constraint on the variable $x$. Note that the expression of $\Pi(x)$ provided by the authors of Ref. [15] is not explicit. In fact, as shown by Eq. (5), it is a functional of $\Pi(x)$. Thus, in practice the explicit form of $\Pi(x)$ has to be established by means of an iteration procedure. It is remarkable, however, that the microcanonical derivation from Ref. [13] results in the same prescription as that of Eq. (5). From the point of view of the issues under discussion here, what matters is the fact that at the end of the iterative procedure $U_{q}$ becomes a well-defined number. Consequently, the resulting expression for $\Pi(x)$ is a simple analytical formula that in the asymptotic limit, $|x|$ $\rightarrow \infty$, with the constraint on the first moment of $|x|$, has the same structure as that derived in Ref. [5],

$$
\Pi(x)=\frac{\widetilde{b}(2-q)}{[1+\widetilde{b}(q-1)|x|]^{1 /(q-1)}} .
$$

Note that the adoption of a constraint on the second moment would lead to

$$
\Pi(x)=\frac{\left[\frac{\widetilde{b}(q-1)}{\pi}\right] \frac{\Gamma(1 /(q-1))}{\Gamma((3-q) /[2(q-1)])}}{\left[1+\widetilde{b}(q-1) x^{2}\right]^{1 /(q-1)}} .
$$

Note that both Eqs. (6) and (7) are the result of an earlier prescription, missing the normalization factor present in Eq. (4). Nevertheless, it has to be pointed out that both Eqs. (6) and (5) share the characteristic of having long tails with Lévy statistics. In fact, it is well known [1] that the anomalous diffusion processes of the Lévy kind in the onedimensional case are characterized by probability distributions $p(x, t)$ whose Fourier transform in the symmetric case reads

$$
\hat{p}(k, t)=\exp \left(-b|k|^{\alpha} t\right),
$$

where $\alpha$ is the Lévy index ranging, in principle, in the interval $0<\alpha \leqslant 2$, and $b$ denotes the diffusion intensity. The inverse Fourier transform of $\hat{p}(k, t)$ of Eq. (8) is characterized by the tail [18]

$$
\lim _{|x| \rightarrow \infty} p(x, t) \propto \frac{t}{|x|^{1+\alpha}},
$$

which would lead immediately to the anomalous entropy index

$$
q=1+1 /(1+\alpha) .
$$

We have to stress, however, that except for the case $\alpha=1$ corresponding to the ballistic limit, the central part of the distribution produced by the Lévy process of Eq. (8) cannot be expressed in an analytical form and can significantly depart from the analytical form of both Eqs. (6) and (5). The adoption of the prescription of Eq. (7) is expected to yield better agreement, but, as we shall see subsequently, it does not seem to be compatible with the nature of the dynamical 


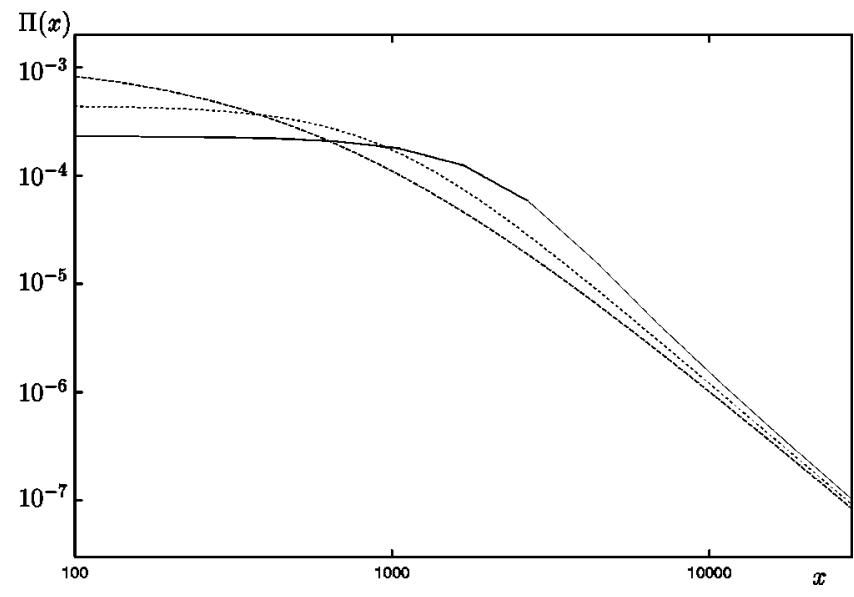

FIG. 1. Comparison of a Lévy distribution (solid line) with distributions obtained by maximizing Tsallis entropy (dashed lines). The Lévy curve is obtained by the inverse Fourier transform Lévy characteristic function with $\alpha=1.5, \quad b=4.4 \times 10^{4}$. The asymptote is proportional to $1 / x^{2.5}$. The long-dashed line comes from Eq. (6) with the constraint on the first moment $(\tilde{b}=0.004, q=1.4)$. The short-dashed line comes from Eq. (7) with the constraint on the second moment $\left(\tilde{b}=1.4 \times 10^{-6}, q=1.8\right)$. The values of the parameter $\tilde{b}$ are selected, in both cases, so as to fit the asymptotic behavior of the Lévy distribution. This constraint results in significant departures from the Lévy distribution at small and intermediate distances.

approach to equilibrium. In Fig. 1 we compare the Lévy distribution to both the prediction of Eqs. (6) and (7) and find that there exists a significant disagreement between Tsallis and Lévy statistics, even though some authors [12] refer to them as equivalent. In this paper we focus our attention on the origin of the difference between the two kinds of statistics. Our dynamic approach leads to a form of equilibrium that is stable, in the sense of the Lévy-Gnedenko theorem [19], while the generalized canonical equilibrium of Eq. (5) is not. This is evident in the free case, due to the difference between the Tsallis and the Lévy structure. In Sec. III we prove that this is so also in the presence of a feedback, with the system of interest, of the generator of fluctuations.

\section{B. From dynamics to thermodynamics}

We are convinced that there are no incontrovertible reasons why the canonical distribution should be the unique form of thermodynamic equilibrium, and on this issue we essentially agree with the point of view of Abe and Rajagopal [13]. However, we are equally convinced that the generalized canonical form of Eq. (5) does not satisfy the stable conditions necessary for Lévy statistics, and the results of the present paper can be thought of as providing plausible evidence of that. To substantiate this view with dynamical arguments, it is convenient to concisely review the results of an earlier work [20].

The ambitious purpose of this earlier work was that of reversing the path from thermodynamics to mechanics established by Boltzmann. The main idea behind Ref. [20] is as follows. The Fokker-Planck equation is a well-known de- scription of the evolution of the probability density in phase space. Many attempts have been made in the literature to derive this equation [21-25]. However, all these attempts rest on the assumption that the bath, responsible for the Brownian behavior of the particle of interest, is given by a set of harmonic oscillators. This means that a statistical assumption must be made on the initial condition of the bath, which is arbitrarily given a canonical equilibrium distribution, corresponding to a given temperature $T$ of the thermal bath.

Consequently, this kind of approach to Brownian motion is only partially dynamical, since significant use is already made of statistical mechanics and thus of thermodynamics [26]. The authors of Ref. [20] adopted a totally different approach. They assumed that a given oscillator, playing the role of a stochastic Brownian particle, interacts with another Hamiltonian system, which should play the role of a bath. They called this second system a booster, to stress that the ensuing approach has to rest only on the dynamical properties of this kind of bath with no use whatsoever of thermodynamical arguments. After establishing the dynamical conditions ensuring the validity of the Fokker-Planck equation, it is found that the oscillator of interest reaches a canonical equilibrium distribution. Due to the nature of the procedure adopted the width of this canonical distribution depends only on the parameters of the Hamiltonian system under study. Consequently, it is possible to derive a mechanical expression for temperature. This is the key result of Ref. [20], which reads

$$
k_{B} T=\left[\frac{\partial}{\partial E} \ln A(E)+\frac{\partial}{\partial E} \ln \left\{\left\langle\xi^{2}\right\rangle_{e q} \operatorname{Re}\left[\hat{\Phi}_{\xi}(\omega)\right]\right\}\right]^{-1} .
$$

Note that $\xi$ denotes the doorway variable, namely, the variable of the booster through which the interaction between the booster and the oscillator of interest is established. The symbol $\hat{\Phi}_{\xi}(\omega)$ denotes the Laplace transform of the correlation function of $\xi$ evaluated at the oscillation frequency of the oscillator. The structure of this expression reflects the application of linear response theory [20]. The correlation function whose Laplace transform is in Eq. (11) is evaluated assuming the booster to be in a microcanonical equilibrium with energy $E$ and this condition is not affected by the interaction with the oscillator. The symbol $A(E)$ denotes the number of states of the booster in the same physical condition, and consequently, obeys the ordinary prescription [27]

$$
A(E) \propto E^{N / 2},
$$

where $N$ is the number of degrees of freedom in the booster.

The authors of Ref. [20] note that for $N \rightarrow \infty$,

$$
\frac{\partial}{\partial E} \ln \left\{\left\langle\xi^{2}\right\rangle_{e q} \operatorname{Re}\left[\Phi_{\xi}(\omega)\right]\right\} \ll \frac{\partial}{\partial E} \ln A(E) .
$$

This means that in the limiting case of infinitely many degrees of freedom the predictions of Boltzmann are recovered: $1 /\left(k_{B} T\right)=(\partial / \partial E)[\ln A E]$. Note that the condition of Eq. (13) holds true if the doorway variable $\xi$ depends on a number of 
particles, which is kept fixed with $N \rightarrow \infty$. In other words, the condition of Eq. (13), or equivalently Boltzmann's principle, rests on an interaction condition, which is crucial for the extensive statistical mechanics perspective to apply.

Let us see this aspect in detail. In Ref. [20] the booster is the the well-known Fermi-Pasta-Ulam (FPU) system [28]. This is a chain of particles interacting with one another via nonlinear interactions. Let us consider two opposite conditions. In the former, which is the one adopted in Ref. [20], the oscillator of interest interacts only with the first particle of the FPU chain. In the latter the oscillator of interest interacts with all the particles of the FPU chain with interaction strength of comparable intensities. The former condition refers to a short-range interaction, confined to the positions of the oscillator of interest and to the first particle of the FPU chain. In the latter condition, the interaction extends over the whole FPU chain. It is evident that the former condition fits the inequality of Eq. (13), whereas the latter does not. This means that the latter condition results in a nonextensive form of dynamics, with a consequent breakdown of the prescriptions of ordinary statistical mechanics. The former condition on the contrary, for $N \rightarrow \infty$, recovers ordinary statistical mechanics. This suggests that the dynamical corrections to the Boltzmann principle, recorded in Ref. [20] for relatively small values of $N$, are a manifestation of incipient nonextensive statistical mechanics, and so are very close to the breakdown of the Fokker-Planck treatment on which the analysis of Ref. [20] rests. Note that when we assign to the oscillator of interest a very low frequency compared to the booster frequencies, the quantity $\operatorname{Re}\left[\hat{\Phi}_{\xi}(\omega)\right]$ turns out to virtually coincide with the time scale $T_{\text {micro }}$ of the variable $\xi$ defined by

$$
T_{\text {micro }} \equiv \int_{0}^{\infty} \Phi_{\xi}(t) d t<\infty .
$$

Herein we focus our attention on the case where

$$
\lim _{t \rightarrow \infty} \Phi_{\xi}(t)=\operatorname{const} / t^{\beta}
$$

with

$$
0<\beta<1 .
$$

The inverse power law form of the correlation function means that we select time memory as the source of violation of extensivity, rather than long-range spatial interactions. The parameter $T_{\text {micro }}$ denotes the correlation time of the fluctuating variable $\xi$. Here we consider the dichotomous case, where the variable $\xi$ has two distinct values $W$ and $-W$ with fluctuating time durations. The correlation function $\Phi_{\xi}(t)$ is proven [29] to be proportional to the second time derivative of the distribution of waiting times in the two states of the variable $\xi$. This function, as we shall see in Sec. IC, depends on another time $\bar{T}$ as well as on $\beta$.

In conclusion, the work of Ref. [20] establishes the dynamical conditions necessary to derive canonical equilibrium. The authors prove that canonical equilibrium implies an interaction with a booster with a finite time scale and a short-range interaction. We can also observe that in this case the use of dynamical arguments yields the same conclusions as the very simple argument based on the law of large numbers. The advocates of the law of large numbers for the foundation of statistical mechanics [30] might judge the dynamical perspective to be of limited use. We prefer to interpret the conclusion of Ref. [20] as proof that in the extensive case the canonical distribution description of thermodynamic equilibrium is unique. The numerical work of Ref. [20] shows that it is very difficult to detect the dynamical corrections to the Boltzmann principle illustrated by Eq. (11) from within an extensive perspective. This is so because the dynamical corrections become significant when the booster is small and as clearly pointed out by the recent work of Gross and Votyakov [31], a small system is essentially nonextensive. Thus, the numerical findings of Ref. [20] refer to a condition very close to the breakdown of the extensive condition and consequently of the canonical equilibrium on which Eq. (11) rests. This means that the dynamical approach can be of great utility. In the case of nonextensive statistical mechanics, the dynamic approach is probably the only nonambiguous way to address the subtle issues posed by the entropic and probabilistic methods.

\section{Stochastic dynamics}

In this section we explain the nature of our dynamical approach to the noncanonical equilibrium distribution. This approach rests on a stochastic method adapted to the need of realizing a dichotomous variable $\xi$ with the two possible values $W$ and $-W$ and with the waiting-time distribution

$$
\psi(t)=\frac{(\beta \bar{T})^{\beta+1}(\beta+1)}{(\beta \bar{T}+t)^{2+\beta}},
$$

where $\beta$ ranges in the interval of Eq. (16). Note that the parameter $\bar{T}$ is of crucial importance to define the time scale of our process and corresponds to the mean residence time in either of the two states of the velocity variable $\xi$. In accordance with the prescriptions of Ref. [29] this waiting-time distribution yields the kind of correlation function $\Phi_{\xi}(t)$ that we plan to study herein [see Eq. (15)]. In fact, as shown in Ref. [29], the form of Eq. (17) yields the correlation function

$$
\Phi_{\xi}(t)=\frac{(\beta \bar{T})^{\beta}}{(\beta \bar{T}+t)^{\beta}},
$$

fitting the asymptotic time limit of Eq. (15). This condition can be considered as the natural one-dimensional counterpart of the two-dimensional billiards of Zaslavsky [32]. From this point of view, our commitment to the adoption of a merely dynamical approach is not broken, since the stochastic approach that will be adopted in Secs. II and III is statistically equivalent to the adoption of the intermittent map of Ref. [33], on which the theoretical work of Ref. [5] is based.

We stress that the equivalence between a dynamical map and a stochastic process is the reason why contact can be established between the dynamical and the entropic approaches. In fact, as shown in Ref. [5] the nonextensive Tsal- 
lis entropic indicator serves the purpose of guessing the most convenient form for the transition probability $\Pi(x)$, which is then related to the waiting-time distribution $\psi(t)$ through the basic property

$$
\Pi(x)=\frac{1}{2} \psi(x / W) / W .
$$

The factor of $1 / 2$ takes into account the fact that the probability of making the jump $x$ is equal to that of making the jump $-x$, namely, the jump in the opposite direction. The authors of Ref. [5] show that the left-hand term of this equality, Eq. (19), can be predicted using entropic arguments, while the right-hand term of the same equation is dictated by dynamical arguments based on the intermittent map of Ref. [33]. These dynamical arguments are supplemented by the assumption of random injection of the trajectory from the chaotic into the laminar region of the intermittent map, an argument leading to an analytical prediction for $\psi(t)$ in complete agreement with the numerical observation of dynamics [33].

The earlier work of Ref. [5] established that the adoption of the method of entropy maximization applied to the nonextensive entropy of Eq. (2) results in a form of $\Pi(x)$, which is compatible with the birth of Lévy statistics. However, the $\Pi(x)$ thus derived is not the equilibrium distribution of the variable $x$. Rather it is the probability for the random walker to make a jump of length $|x|$. This is a stationary property determined by the special kind of booster under study here. The ensuing diffusion process yields a Lévy form as a result of the Lévy-Gnedenko theorem [19]. As shown in Fig. 1, the shape of this distribution departs from the form of the generalized canonical distribution of Eq. (5), even if we adopt the constraint on the second moment, in spite of the fact that it does not fit the nature of the dynamical approach illustrated here.

To account for this discrepancy we might make the conjecture that the comparison between the Lévy statistics and the Tsallis generalized canonical distribution is not appropriate. The former refers to a diffusion process and the latter to an alleged equilibrium condition. Actually, after exploring this possibility we shall conclude that the latter, at least in the case of the dynamic model of the present paper, cannot reflect an equilibrium property. However, at the present stage, we are forced to develop a picture comparable to that of the earlier work of Ref. [20]. We have to study a case where the variable $x$ not only undergoes the influence of the diffusion producing fluctuations, but it produces a feedback on its own "bath," balancing the diffusion process so as to create an equilibrium condition. This is the condition to compare to the generalized canonical distribution of Eq. (5). In other words, to address, from a dynamical perspective, the issues recently dealt with by Abe and Rajagopal [13] we cannot disregard the feedback from the system to the "bath."

\section{Purpose of the present paper}

At this stage it is much easier for us to illustrate the main purpose of the present paper. We want to explore a condition where the booster does not fulfill the key condition of Eq. (14), that is, the microscopic relaxation time diverges, and we want to assess whether or not this divergence leads to the generalized canonical distribution of Eq. (5). We aim at answering the question: Which is the form of the equilibrium distribution reached by the system of interest when the time scale of the booster is infinite?

To answer this question we do not adopt, as done in Ref. [20], a Hamiltonian approach. This approach is difficult for obvious reasons. A numerical simulation check of the theoretical prediction would imply technical difficulties caused by the slowness of the booster itself. This means that the anomalous booster is replaced, as done in earlier papers $[34,35]$, by a generator of a dichotomous fluctuation with an inverse power law distribution of waiting times. The essential ingredient of the approach of Ref. [20], not yet present in the dynamical derivation of a free Lévy diffusion, is a feedback of the diffusing variable on the generator of the fluctuations. This aspect has already been considered in the earlier work of Ref. [34]. However, in that paper the dynamical approach to the feedback was replaced by a phenomenological friction, which did not allow the authors of that paper to keep the promise of resting solely on dynamical arguments at any level. In conclusion, we adopt the program of Ref. [20], based on the observation of the fluctuation-dissipation process caused by the interaction of a particle with a booster having no finite time scale. The presence of feedback serves the purpose of balancing the diffusion process with dissipation so as to result eventually in an equilibrium condition.

With the program of Ref. [20] in mind, we have to refer ourselves to the correlation function, Eq. (18), with the index $\beta$ fulfilling the condition of Eq. (16), and so implying the breakdown of the time-scale separation between the macroscopic and the microscopic levels. The realization of the program of statistical mechanics requires an accurate definition of the process of memory erasure associated with the transition from one to the other velocity state [36]. This is more conveniently defined by the waiting-time distribution $\psi(t)$ of Eq. (17), than by the correlation function of Eq. (18). We see that even if the condition of Eq. (16) applies, the time $\bar{T}$ $=\int_{0}^{\infty} t \psi(t) d t$ remains finite. Memory of microscopic dynamics is lost in times $t \gg \bar{T}$.

At this stage it is convenient to support our claim concerning memory erasure with arguments borrowed from the earlier investigation of Gaspard and Wang [37]. These authors studied the Kolmogorov complexity of the Manneville map and found that in the regime corresponding to the dynamical foundation of Lévy processes, the Kolmogorov complexity is a linear function of time. This means that at a given time $t$ the number of transitions $M$ from the one to the other laminar region is given by $M \propto t / \bar{T}$. This means that for $t \rightarrow \infty$ the conditions for the realization of the generalized version of the central limit theorem are fulfilled, since the function defined by

$$
p(x, M) \equiv \Pi(x) * \Pi(x) * \ldots * \Pi(x) * p_{\text {in }}(x),
$$

where the asterisk denotes a convolution, for $M \rightarrow \infty$ tends to the Lévy distribution. One might be tempted to make the 
conjecture that in the presence of feedback the number $M$ cannot increase beyond some limit, and that equilibrium is reached with a relatively low value of $M$ so as to allow the distribution to maintain the structure of a generalized canonical distribution in the manner of Tsallis. In this paper we limit our analysis to the case where the linear response theory of Sec. III holds true. This forces us to adopt a feedback so weak that this possibility does not emerge from our simulations.

\section{DYNAMICAL MODEL}

The dynamical model studied here depends on a process of free diffusion with feedback, established through control of a dynamical parameter of the booster. This feedback process has the role of balancing diffusion so as to realize dynamically the equilibrium condition. We illustrate first the dynamical model used as the generator of free diffusion, then we show how the feedback is realized, and finally we illustrate the numerical technique adopted.

\section{A. Free diffusion}

Formally free diffusion is realized using the equation of motion

$$
\frac{d x(t)}{d t}=\xi(t)
$$

Here the dynamical variable $x(t)$ denotes either a spatial coordinate or a velocity. In the former case the variable $\xi(t)$ has to be considered a fluctuating velocity, while in the latter case it is regarded as a fluctuating acceleration. The results are equivalent, and the reader can adopt either of them, even if we consider $x(t)$ to be a spatial coordinate so as to make the connection with earlier work [5] more natural.

We assume the variable $\xi(t)$ to be dichotomous, namely, we shall assign to this variable only two distinct values, either $W$ or $-W$. The motivation for this choice is not only simplicity, but also has to do with the main purpose of this paper, that of assessing the specific form the noncanonical equilibrium, and also of course, and prior to this purpose, that of proving that deviations from canonical equilibrium can be generated as a consequence of the breakdown of the ordinary condition of time-scale separation between macroscopic and microscopic processes. In the limiting case where the variable $\xi(t)$ is Gaussian, and consequently produces a Gaussian equilibrium distribution [38], the resulting diffusion process is Gaussian and the final equilibrium distribution can turn out to be Gaussian as well [34], as a result of having established a seed of ordinary statistics at the microscopic level. For this reason the choice of microscopic statistics, departing from the ordinary statistical condition is crucial, and the adoption of the dichotomous assumption serves the purpose of eventually establishing a noncanonical equilibrium.

The variable $\xi(t)$ keeps one of the two possible values for times with a random duration. Thus, a statistical treatment must be adopted and a waiting-time distribution $\psi(t)$ is used. The time intervals of sojourn in a given state are labeled by an integer index $k$ running from 1 to $+\infty$. At the end of any sojourn the variable can either change its value, and thus make a transition from $W$ to $-W$ or from $-W$ to $W$, or it can keep its original value. The probability of changing values and that of keeping the same value are equal, and thus equal to $1 / 2$. We can interpret the variable $\xi(t)$ as a function of the continuous time $t$ by adopting the following prescription:

$$
\xi(t)=\sum_{k} \xi_{k} \chi_{t_{k}, t_{k+1}}(t),
$$

where the times $t_{k}$ and $t_{k+1}$ denote the beginning and the ending time of the $k$ th sojourn, respectively, and the values $\xi_{k}$ are randomly assigned either the value $W$ or the value $-W$ with equal probability and $\chi_{t_{k}, t_{k+1}}(t)=1$ if $t_{k} \leqslant t$ $<t_{k+1}$. The sequel of the times $t_{k}^{\prime}$ is fixed by the waitingtime density distribution $\psi$ by selecting a given value $\tau_{k}$ of the interval $\left[\tau_{k}, \tau_{k}+\epsilon\right]$ with $\epsilon \ll 1$ with the probability $\psi\left(\tau_{k}\right) \epsilon$. The waiting-time distribution $\psi$ is assigned an inverse power law form determined by the constraint of yielding the correlation function

$$
\Phi_{\xi}(t)=\frac{(\beta \bar{T})^{\beta}}{(\beta \bar{T}+t)^{\beta}},
$$

where $\beta$ is a positive number determining the integrability of the correlation function. If $\beta>1$, the correlation function, Eq. (23), is integrable and the microscopic time scale

$$
T_{\text {micro }} \equiv \int_{0}^{\infty} \Phi_{\xi}(t) d t=\frac{\beta \bar{T}}{\beta-1}
$$

can be defined. According to Refs. [39,29] the form of $\psi(t)$ is determined by that of $\Phi_{\xi}(t)$ through the relation

$$
\psi(t)=\bar{T} \frac{d^{2}}{d t^{2}} \Phi_{\xi}(t),
$$

which yields

$$
\psi(t)=\frac{(\beta+1)(\beta \bar{T})^{\beta+1}}{(\beta \bar{T}+t)^{\beta+2}} .
$$

We note that the parameter $\bar{T}$ appearing in both Eqs. (23) and (26) is the mean sojourn time. Thus, in some sense, when the microscopic time of Eq. (24) becomes infinite, the role of the microscopic time scale is played by $\bar{T}$.

By integration of Eq. (21) we get

$$
x(t)=\xi_{n}\left(t-t_{n-1}\right)+\sum_{k=0}^{n-1} \xi_{k} \tau_{k},
$$

where $t$ is a time located in the interval $\left[t_{n-1}, t_{n-1}+\tau_{n}\right]$ with $t_{n-1}=\sum_{k=0}^{n-1} \tau_{k}$, in accordance with the earlier prescriptions. Furthermore, the transition probability of Sec. IA is obviously related to the waiting-time distribution of Eq. (19). Note that, as discussed in detail in Refs. $[34,35,40]$, there are 
two distinct basins of attraction for the diffusion process resulting from the repeated occurrence of the transition process of Eq. (19), the Gauss basin for $\beta>1$ and the Lévy basin for $\beta<1$.

\section{B. The booster linear response and diffusion control}

To control diffusion in such a way so as to generate a known equilibrium distribution we adopt a model based on replacing Eq. (21) with

$$
\frac{d x(t)}{d t}=\xi_{x}(t)
$$

which means that the motion of the fluctuating variable $\xi_{x}(t)$ also depends on the value of the dynamic variable $x$. We make the simplifying assumption that the relaxation time or response time of the booster, $T_{B}$, is shorter than that of the particle of interest, $T_{R}$, so as to ensure for the booster a time dependent equilibrium condition determined by the state of the system of interest. In other words, the booster is assumed to be in a condition of equilibrium determined by the variable $x$. As the variable $x$ moves from an initial condition $x(0)$ out of equilibrium state, i.e., from a position larger than the fluctuations of $x$ itself, to the final equilibrium, the booster correspondingly moves from an equilibrium to another equilibrium condition. All this is illustrated by the following mathematical arguments. These arguments do not aim at providing a rigorous mathematical treatment, but rather a heuristic treatment reflecting the physical condition that we are assigning to the booster.

To make the analysis in terms of a strictly stochastic process, we build up the following regularized process $\tilde{x}_{T_{\rho}}(t)$, defined by

$$
\begin{aligned}
\frac{d \tilde{x}_{T_{\rho}}(t)}{d t} & \equiv \frac{x(t)-x\left(t-T_{\rho}\right)}{T_{\rho}}=\frac{1}{T_{\rho}} \int_{t-T_{\rho}}^{t} \xi_{x}\left(t^{\prime}\right) d t^{\prime} \\
& \equiv \frac{1}{T_{\rho}} \int_{t-T_{\rho}}^{t} \xi_{\tilde{x}}\left(t^{\prime}\right) d t^{\prime},
\end{aligned}
$$

where $T_{\rho}$ is a regularization time and in the last equivalence it has been explicited the spatial dependence of the noise on $\tilde{x}$. This new process $\tilde{x}$ has the characteristics of $x(t)$ in the mean, but its fluctuations are smoothed by using the temporal mean.

If the response time $T_{B}$ of the bath is fast and if $T_{\rho}$ is long enough, but always smaller than relaxation time $T_{R}$ of the variable $x$, one can suppose that the phase space mean is almost equivalent to the temporal mean,

$$
\frac{d \tilde{x}_{T_{\rho}}(t)}{d t}=\frac{1}{T_{\rho}} \int_{t-T_{\rho}}^{t} \xi_{\tilde{x}}\left(t^{\prime}\right) d t^{\prime} \sim\left\langle\xi_{\tilde{x}}\right\rangle .
$$

Now, assuming linear response is valid, the mean value of the thermal bath variable depends on $\tilde{x}$ as

$$
\left\langle\xi_{\tilde{x}}\right\rangle=-\gamma \tilde{x}
$$

With the linear response hypothesis inserted into Eq. (30) the equation for the regularized process

$$
\frac{d \tilde{x}_{T_{\rho}}(t)}{d t} \sim-\gamma \tilde{x}_{T_{\rho}}(t)
$$

is obtained. The solution $\tilde{x}_{T_{\rho}}(t)=x(0) e^{-\gamma t}$ shows a relaxation toward the equilibrium $x \simeq 0$, on a time scale $T_{R} \simeq 1 / \gamma$.

The analogy with a stochastic equation, such as the Ornstein-Uhlenbeck or its extension to Lévy noise [41], is completed by adding a fictitious stochastic noise $d \widetilde{\xi}_{T_{\rho}}(t)$ in Eq. (32) that summarizes the fluctuations on the time scale $T_{\rho}$ and that compensates for the approximation inserted into Eq. (30),

$$
d \tilde{x}_{T_{\rho}}(t)=-\gamma \tilde{x}_{T_{\rho}}(t) d t+d \widetilde{\xi}_{T_{\rho}}(t) .
$$

Hence, with the assumptions of linear response theory and the relaxation times of the thermal bath faster than of that of the variable of interest (partial equilibrium), a diffusive process becomes an equilibrium process. We emphasize that the analogy (33) is true only if

$$
T_{B} \ll T_{\rho} \ll T_{R}=\gamma^{-1} \text {. }
$$

By using this simplified analysis it is also possible to make qualitative predictions about the equilibrium distribution.

When $x \simeq 0$, the feedback $-\gamma \tilde{x}_{T_{\rho}}(t)$ can be neglected with respect to the fluctuations $\widetilde{\xi}_{T_{\rho}}(t)$. This limit corresponds to free diffusion for which the central limit theorem (generalized or not) can be used. The distribution is Gaussian or Lévy's according to whether the correlation function is integrable or not.

When $|x| \gg 0$ the feedback term (31) prevails over fluctuations in Eq. (33). Then the dynamical variable is pushed back to values near the equilibrium $x \sim 0$ as an attractive field quenching the diffusion. This description corresponds, at least for the region $|x| \ll W / \gamma$, to what one could obtain if in Eq. (33) the fictitious noise $\widetilde{\xi}_{T_{\rho}}(t)$ is simply stochastic [41].

The condition (31) is satisfied when the time evolution of $\xi_{x}$ is replaced by the stochastic variable $\xi_{k}$ appearing in Eq. (22) with the the stochastic variable $\xi_{k}\left(x_{k}\right)$, which has two distinct values with the same probability,

$$
\xi_{k}\left(x_{k}\right) \equiv\left\{\begin{array}{c}
W_{+}\left(x_{k}\right)=W-\gamma x_{k}, \\
W_{-}\left(x_{k}\right)=-W-\gamma x_{k} .
\end{array}\right.
$$

In the time scale $T_{\rho}$, we can replace the variable $\xi_{x}(t)$ of Eq. (28) with its average over the faster bath fluctuations, and thus with

$$
\left\langle\xi_{x}\right\rangle=\frac{1}{2}[W-\gamma x-(W+\gamma x)]=-\gamma x .
$$

In Fig. 2 we show a sample trajectory corresponding to the dynamic prescriptions of Eq. (35). In the lateral region, 


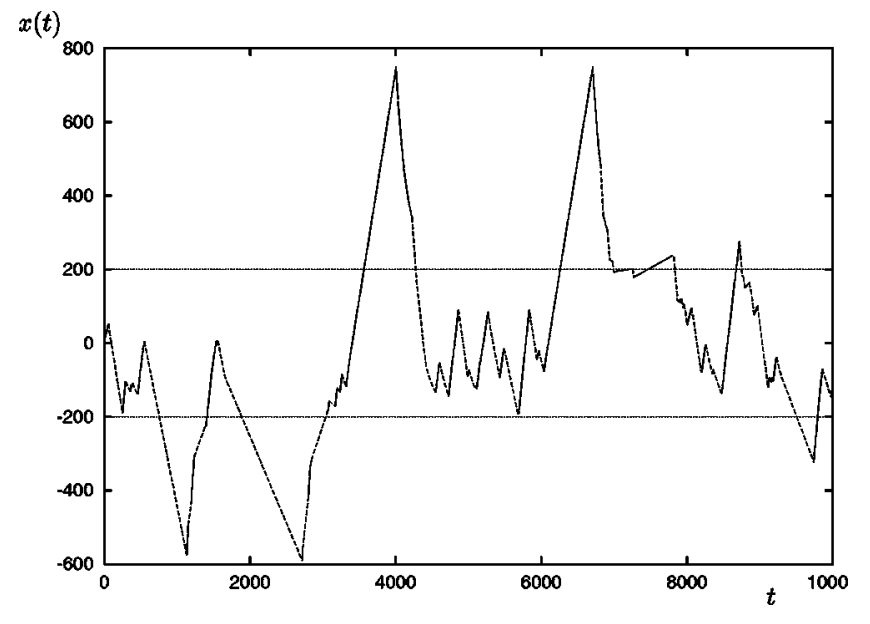

FIG. 2. Sample trajectory for the diffusion process with feedback defined in Eq. (28). The solid and dashed lines denote the states $W_{+}$and $W_{-}$, respectively. See Eq. (35) for the definition of these two states. The two horizontal lines defining the central stripe correspond to the levels $W / \gamma$ and $-W / \gamma$. The parameters used have the values $\gamma=5 \times 10^{-3}, \quad W=1, \quad \beta=0.5, \quad \bar{T}=50$.

after the end of the state that has pushed the trajectory outside the central region, the slope assigned to the trajectory by the ensuing states have always the same sign, either negative for the top region or positive for the bottom region. This is a consequence of the definition itself of side regions and central stripe, the former being those characterized by $|x|$ $>W / \gamma$ and the latter referring to $|x|<W / \gamma$. The final effect is that the trajectories that have exited the central region are steadily brought back to it. We see that, on the contrary, in the central region the trajectory fluctuates back and forth more or less as it would do in the case of free diffusion. This is perhaps the intuitive reason why the linear response theory of Sec. III, referring to a central region of extremely large size, makes the system's statistics fall in the Lévy basin of attraction as it does in the free case [35].

\section{Computer simulation}

The computer simulations are done generating the trajectories of Eq. (27) with the assumption that the values $W_{+}\left(x_{k}\right)=W-\gamma x_{k}$ and $W_{-}\left(x_{k}\right)=-W-\gamma x_{k}$ have the same probability. The distribution of sojourn times in one of these two states is given by the function $\psi(t)$ of Eq. (26), which, in turn, is realized by a suitable nonlinear transformation of a variable with a uniform distribution in the interval $[0,1]$ obtained by a standard routine for the generation of random numbers. It has to be pointed out that the value of either $W_{+}\left(x_{k}\right)$ or $W_{-}\left(x_{k}\right)$ is determined by the value that the variable $x$ has at the moment when a new state is established and it is kept fixed until the end of the sojourn in this state, namely, its value is fixed in the interval $\left[t_{k}, t_{k+1}\right]$. Each trajectory is generated according to the integral of Eq. (28) with the initial condition $x(0)=0$ and it is observed until the time $T_{f} \equiv 20 / \gamma \gg T_{R}$. At this time the value is recorded in the form of a histogram. The distribution of the variable $x$ at this final time, assumed to correspond to equilibrium, is obtained by generating a sufficiently large number of trajectories and constructing a histogram.

\section{THEORETICAL PREDICTION}

We are now in a position to demonstrate that in the case of extremely weak feedback the resulting equilibrium distribution is Lévy. There are no compelling theoretical predictions in the case of strong feedback, except the heuristic arguments used in Sec. I.

According to the strategy adopted in Ref. [20] the theoretical approach to friction implies the treatment of the response of the booster to an abruptly applied external perturbation. Using the notation adopted in Ref. [20], this has to do with the solution to the equation of motion

$$
\frac{\partial}{\partial t} \rho(\xi, t)=\Gamma(K) \rho(\xi, t),
$$

where $\Gamma(K)$ denotes the operator driving the motion of the probability distribution $\rho(\xi, t)$ in the presence of a perturbation of intensity $K$. We assume that this operator can be expressed as the sum of an unperturbed and a perturbed part,

$$
\Gamma(K)=\Gamma_{0}+K \Gamma_{1},
$$

where $\Gamma_{0}$ denotes the operator driving the unperturbed motion of the variable $\xi$ and $K \Gamma_{1}$ denotes the operator corresponding to the perturbation. The intensity of this perturbation can be freely changed by modifying the size of the parameter $K$. We apply the perturbation abruptly at time $t$ $=0$ to the booster, assumed to be in the equilibrium state $\rho_{e q}$. The perturbation strength is assumed to be so weak as to make it possible to solve Eq. (37) at the first-order perturbation,

$$
\frac{\partial}{\partial t} \rho_{1}(\xi, t)=\Gamma_{0} \rho_{1}(\xi, t)+K \Gamma_{1} \rho_{0}
$$

where $\rho_{0}$ and $\rho_{1}$ denote the zeroth- and the first-order distribution density, respectively. We assume that the system before the abrupt perturbation is in thermodynamic equilibrium. This means that we identify $\rho_{0}$ with $\rho_{e q}$, which is assumed to fulfill the following equation:

$$
\Gamma_{0} \rho_{e q}=0 .
$$

The formal solution to Eq. (39) is

$$
\begin{aligned}
\rho_{1}(\xi, t) & =K \int_{0}^{t} d t^{\prime} \exp \left[\Gamma_{0}\left(t-t^{\prime}\right)\right] \Gamma_{1} \rho_{0}\left(\xi, t^{\prime}\right) \\
& =K \int_{0}^{t} d t^{\prime} \exp \left(\Gamma_{0} t^{\prime}\right) \Gamma_{1} \rho_{0}\left(\xi, t-t^{\prime}\right) \\
& =K \int_{0}^{t} d t^{\prime} \exp \left(\Gamma_{0} t^{\prime}\right) \Gamma_{1} \rho_{e q}
\end{aligned}
$$


We assume that at equilibrium the mean value of $\xi$ vanishes. As a consequence, Eq. (41) yields

$$
\langle\xi(t)\rangle=K \int_{0}^{t} C\left(t^{\prime}\right) d t^{\prime}
$$

where

$$
C(t) \equiv\left\langle\xi \exp \left(\Gamma_{0} t\right) \Gamma_{1}\right\rangle_{e q} .
$$

This concise expression means a trace over $\xi$ of the distribution obtained by applying to $\rho_{e q}$ first the perturbation operator $\Gamma_{1}$, then the unperturbed time evolution operator $\exp \left(\Gamma_{0} t\right)$ and finally the variable $\xi$ itself.

In the case of ordinary statistical mechanics one usually makes the assumption that the variable $\xi$ has Gaussian statistics. This means a continuous variable driven by both the unperturbed stochastic environment and by the perturbation of intensity $K$. To define the action of the perturbation on the time evolution of the $\xi$ trajectory, let us switch off the influence of the stochastic environment. In this case the perturbation action would be expressed by $d \xi / d t=K$. Moving from the Heisenberg-like to the Schrödinger-like representation, we would obtain $(\partial / \partial t)[\rho(\xi, t)]=K(\partial / \partial \xi)[\rho(\xi, t)]$, which implies that the perturbation operator is

$$
\Gamma_{1}=\frac{\partial}{\partial \xi} .
$$

Note that in the Gaussian case $\rho_{e q}(\xi)$ is a Gaussian distribution proportional to $\exp \left[-\xi^{2} /\left(2\left\langle\xi^{2}\right\rangle_{e q}\right)\right]$. Consequently $C(t)$ of Eq. (43) becomes

$$
C(t)=\Phi_{\xi}(t) .
$$

Note that

$$
d\langle x(t)\rangle / d t=\langle\xi(t)\rangle
$$

and that, in the absence of perturbations, with all the trajectories starting at $x=0$,

$$
\left\langle x^{2}(t)\right\rangle_{0}=2\left\langle\xi^{2}\right\rangle_{e q} \int_{0}^{t} d t^{\prime} \int_{0}^{t^{\prime}} d t^{\prime \prime} \Phi_{\xi}\left(t^{\prime \prime}\right) .
$$

We adopt the subscript 0 to indicate that the time evolution of the second moment takes place in the absence of perturbations. Using Eqs. (46), (47), and (42) supplemented by Eq. (45) and setting equal to zero an arbitrary integration constant so as to ensure a response proportional to the perturbation strength, we arrive at

$$
\langle x(t)\rangle=K\left\langle x^{2}(t)\right\rangle_{0} /\left(2\left\langle\xi^{2}\right\rangle_{e q}\right) .
$$

This equation is a generalized Einstein relation [42], more recently discussed by Barkai and Fleurov [43].

It is evident that the ordinary condition (48) cannot apply in the case under study here corresponding to the conditions (15) and (16). In fact, in this latter case the adoption of the ordinary linear response relation would yield an infinite current. Earlier investigation [44] has established that the ordi- nary linear response theory breaks down and a new form of linear response to perturbation shows up, which, however, cannot be expressed in terms of unperturbed dynamics. This means that the response depends on the dynamical model adopted and must be established through numerical integration.

In conclusion, using the strategy of Ref. [20] we find that in the presence of feedback the free diffusion process

$$
d x / d t=\xi
$$

must be replaced with

$$
d x / d t=\langle\xi(t)\rangle_{x}+\xi
$$

where we have divided the current, in accordance with linear response theory into the sum of two terms. The zeroth-order term is given by the fluctuation $\xi$ assumed to be in the same unperturbed condition as in Eq. (49). The first-order term is $\left\langle\xi(t)_{x}\right\rangle$. Although the variable $\xi$ sojourns for long times in one of the two-velocity states, we assume the feedback to be so weak as to be compatible with $\xi$ making many jumps from one velocity state to the other while the value of the variable $x$ remains essentially unchanged. On the other hand, setting [20]

$$
K=-\Delta^{2} x
$$

where $\Delta^{2}$ is a constant, we obtain

$$
d x / d t=-\gamma x(t)+\xi(t)
$$

and

$$
\gamma=\Delta^{2} \chi
$$

the susceptibility $\chi$ being defined by

$$
\chi \equiv \int_{0}^{\infty} C(t) d t .
$$

We are now in a position to address the problem under discussion here via three approximation steps.

(i) We replace Eq. (52) with

$$
d x / d t=-\gamma x(t)+\eta(t),
$$

where $\eta$ is a Lévy noise [41]. This means that the variable $\eta$ in one single time step produces jumps proportional to those produced by the variable $\xi$ sojourning for a time $t=\eta / W$ in one of the two velocity states $[34,35]$.

(ii) We make a numerical simulation of Eq. (52).

(iii) We make a completely dynamical treatment of the whole fluctuation-dissipation process.

We shall refer to approximation (i) as the stochastic approximation. The advantage of this approximation is that Eq. (52) is made equivalent to an equation studied years ago by West and Seshadri [41].The stochastic force results in a phase space operator equivalent to a fractional derivative of order $\alpha$, with

$$
\alpha=\beta+1 \text {. }
$$


Thus, it is possible to show [41] (see also Refs. [36,35] for a more recent discussion of the same problem) that the Fourier transform of the distribution density $\sigma(x, t), \hat{\sigma}(k, t)$ reads

$$
\frac{\partial}{\partial t} \hat{\sigma}(k, t)=-\left(b|k|^{\alpha}+\gamma k \frac{\partial}{\partial k}\right) \hat{\sigma}(k, t),
$$

which yields the following characteristic function

$$
\ln \hat{\sigma}(k, t)=-\frac{\left(1-e^{-\alpha \gamma t}\right)}{\alpha \gamma} b|k|^{\alpha},
$$

which for no dissipation has the familiar form

$$
\lim _{\gamma \rightarrow 0} \ln \hat{\sigma}(k, t)=-t b|k|^{\alpha} .
$$

However, asymptotically, in time we obtain the equilibrium condition

$$
\hat{\sigma}(k, \infty)=\exp \left(-\frac{b|k|^{\alpha}}{\alpha \gamma}\right)=e^{-b_{\gamma}|k|^{\alpha}} .
$$

The second equality defines the equilibrium parameter $b_{\gamma}$ of the Lévy characteristic function. Approximation (ii) is referred to as the dissipative Lévy walk, and has already been discussed in an earlier work [34,35], where it was shown that in the limiting case of $\gamma \rightarrow 0$ it leads to an equilibrium distribution equivalent to that of Eq. (60).

\section{THE NUMERICAL SIMULATION}

An interesting result of the numerical simulation of the dynamical model is that the equilibrium distribution is not uniquely determined. The condition of weak feedback yields a distribution that has properties different from those of the equilibrium distribution stemming from the condition of strong feedback.

The numerical simulation of the dynamical model can be done both in the case of weak and strong feedback. Interesting new effects are revealed by the simulation of the case of strong feedback. However, these are left as a subject for future theoretical discussions. Here we illustrate only the simulation results concerning the case of weak feedback. According to the program of Ref. [20], we have developed a theory resting on the linear response theory, although in the nonconventional form of Sec. III and consequently on the assumption of a very weak feedback. Therefore, the simulation results here discussed refer to a case of feedback so weak as to ensure that the requirements of Sec. III are fulfilled.

Figure 3 refers to the Lévy basin of attraction. We notice that the dissipative Lévy walk results in pronounced peaks. These peaks are produced by the fact that the trajectories cannot overcross the values $x= \pm W / \lambda$. These peaks signal the region within which a good agreement among the fully dynamic treatment, the dissipative Lévy walk, and the stochastic approximation is expected. Note that, as remarked in Sec. III, the stochastic approximation is equivalent to the equilibrium of the theory of West and Seshadri (WS) [41],

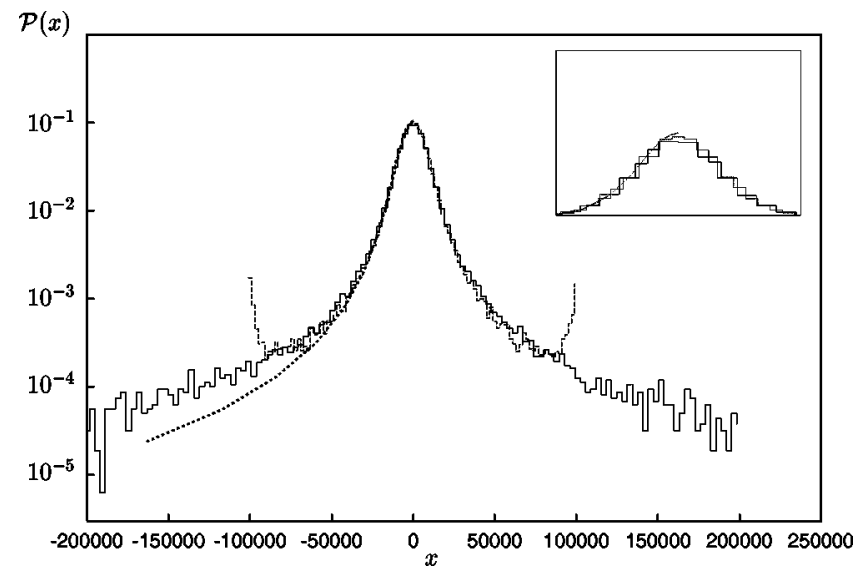

FIG. 3. The equilibrium distribution in the Lévy's basin of attraction. The full line histograms refer to the numerical simulation with $\beta=0.5, \gamma=10^{-5}, W=1, \bar{T}=50$. We set the bin size equal to 2000 . The dashed line histograms illustrate the result of the numerical simulation of the dissipative Lévy walk of Eq. (52). The prediction of the stochastic approximation, or equivalently of the WS statistics, is denoted by means of the heavily dashed line. To make the figure less heavy we plot only the left part of the distribution predicted by the WS statistics. The WS equilibrium is obtained by evaluating the inverse Fourier transform of Eq. (60) with $b_{\gamma}$ $=417771, \alpha=1.5$, and $\gamma=10^{-5}$. This is the value of $b_{\gamma}$ that according to the theoretical prediction of Eq. (61) corresponds to the parameters of the dynamical treatment. The inset shows, for clarity, the enlarged portion of the figure corresponding to the $x$-axis interval $[-20000,20000]$. The enlargement of the ordinates is done after conversion to a linear scale.

where the parameter $b_{\gamma}$ of Eq. (60) is calculated with the following formula [35]:

$$
b_{\gamma}=\frac{W(\beta \bar{T} W)^{\beta}}{\gamma(\beta+1)} \sin \left(\frac{\pi}{2} \beta\right) \Gamma(1-\beta),
$$

where $\bar{T}$ and $\beta$ are defined in Eq. (23) and $\Gamma(\cdot)$ is the Euler gamma function. We see that in the region enclosed by the peaks a very good accordance among these three distinct approaches is indeed found.

Figure 4 illustrates the situation in the Gauss basin of attraction. In this case the side peaks of the dissipative walk disappear. The counterpart of the WS statistics here is the Ornstein-Uhlenbeck statistics [41], already used in an earlier publication [35] to predict the form of equilibrium distribution in the Gauss basin of attraction. We use the same prediction where the variance $\sigma^{2}$ is

$$
\sigma^{2}=\frac{\beta W^{2} \bar{T}}{\gamma(\beta-1)}
$$

and we find that the agreement with the other two treatments is again very good.

We are convinced that the simulation results of Fig. 3 are a reliable numerical evidence of the fact that the dynamic approach to noncanonical equilibrium yields the analytical form proposed 18 years ago by West and Seshadri [41], here 


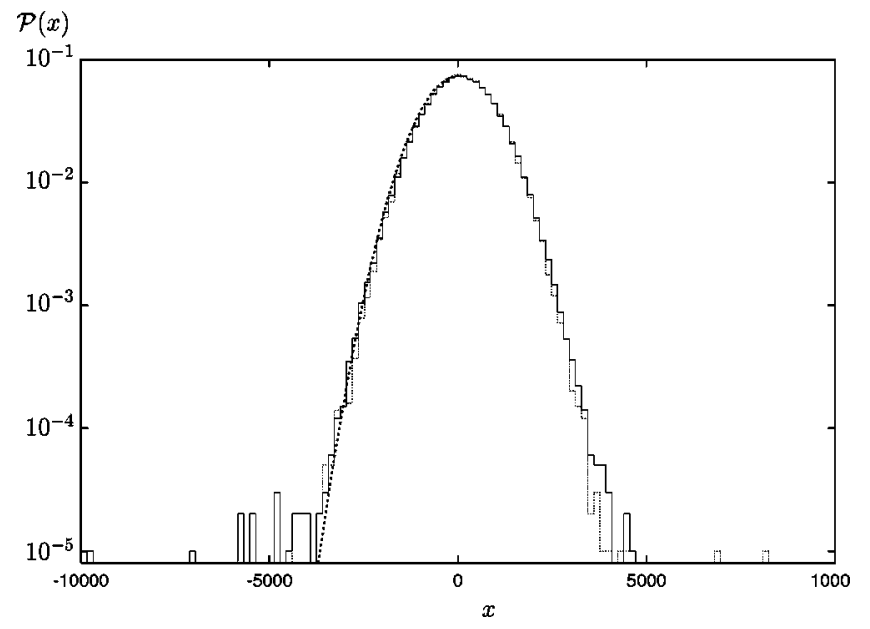

FIG. 4. The equilibrium distribution in the Gaussian basin of attraction. The full line histograms refer to the numerical simulation with $\beta=3, \gamma=10^{-4}, W=1, \bar{T}=50$. We set the bin size equal to 120. The dashed line histograms refer to the numerical solution of the Gaussian counterpart of Eq. (52). The heavily dashed line is the theoretical prediction of the Ornstein-Uhlenbeck process with $\sigma^{2}$ $=7.5 \times 10^{5}$ calculated from Eq. (62). To make the figure less heavy we plot only the left portion of this theoretical prediction.

referred to as WS statistics. However, Tsallis statistics and WS statistics have in common the analytical shape of the tails, which is an inverse power law. This might suggest that the accordance between our simulation results and Tsallis statistics is as satisfactory as, or more satisfactory than, the accordance with the WS statistics. We now show that it is not so, and that the accordance of our results with Tsallis statistics is much less satisfactory than with the WS statistics. According to the spirit of our dynamic approach, the comparison should be done with the Tsallis form of equilibrium corresponding to a constraint on the first moment of $|x|$. This would produce a more significant departure from the WS statistics, and from our simulation results, the WS statistics being, in fact, a Lévy form of equilibrium statistics. The reader can easily be convinced about this property by observing Fig. 1. Thus, we decided to discuss the comparison between our simulation results and Tsallis statistics adopting a condition more favorable to the Tsallis statistics, namely, the analytical proposal of Eq. (7).

In Fig. 5 we establish an exact accordance between Tsallis and WS statistics in the region of large distances. We see that the WS statistical prescription yields a very satisfactory accordance with the results of simulation also in the central region, while the Tsallis noncanonical equilibrium does not. In Fig. 6 we organize the comparison in such a way as to produce the best fitting between Tsallis statistics and our simulation in the central part of the equilibrium distribution. We see that this has the effect of making the discrepancy between Tsallis statistics and WS statistics much worse in the region of large distances. We see that our simulation results are much closer to the WS equilibrium than to the Tsallis equilibrium. We have to point out, furthermore, that the region of large distances in Fig. 6, where the Tsallis form

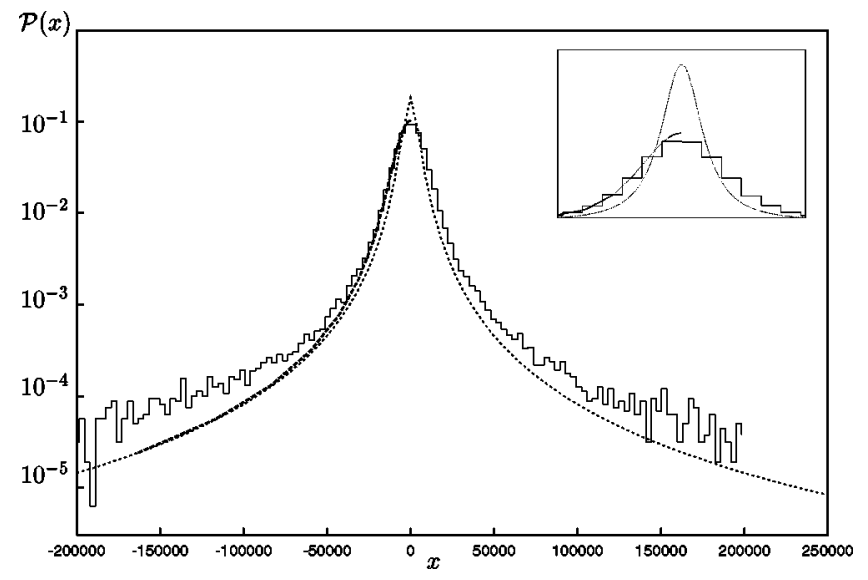

FIG. 5. Tsallis and WS statistics versus the numerical results. The WS equilibrium is denoted by the heavily dashed line and, as in Fig. 3, only the left part of it is illustrated. It corresponds to the inverse Fourier transform of the distribution of Eq. (60) with $b_{\gamma}$ $=417771, \alpha=1.5$, and $\gamma=10^{-5}$. The parameter $b_{\gamma}$ was found as in Fig. 3. The Tsallis equilibrium, corresponding to the proposal of Eq. (7), is illustrated by the dotted line. The dotted line is plotted so as to coincide with the heavily dashed line in the region of large distances $\left(\tilde{b}=6 \times 10^{-8}, q=1.8\right)$. The inset shows, for clarity, the enlarged portion of the figure corresponding to the $x$-axis interval $[-20000,20000]$. The enlargement of the ordinates is done after conversion to a linear scale.

of noncanonical equilibrium apparently yields a better agreement with our numerical results, is probably a nonstationary region, which depends on the observation time. We do not have yet any theory concerning this region, but we have the result of the numerical observation. The numerical simula-

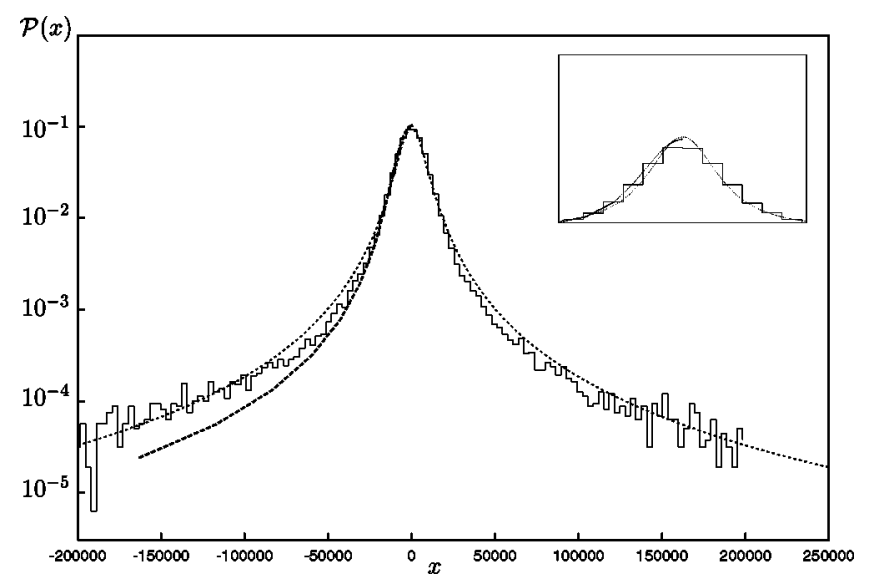

FIG. 6. Tsallis and WS statistics versus the numerical results. The WS equilibrium is denoted by the heavily dashed line and, as in Fig. 3, only the left part of it is illustrated. It corresponds to the inverse Fourier transform of the distribution of Eq. (60) with $b_{\gamma}$ $=417771, \alpha=1.5$, and $\gamma=10^{-5}$. The Tsallis equilibrium, corresponding to the proposal of Eq. (7), is illustrated by the dotted line $\left(\widetilde{b}=2 \times 10^{-8}, q=1.8\right)$. The dotted line is plotted so as to get the best fitting with the heavily dashed line in the central region. The inset shows, for clarity, the enlarged portion of the figure corresponding to the $x$-axis interval $[-20000,20000]$. The enlargement of the ordinates is done after conversion to a linear scale. 
tion shows that this region triggers nonstationary effects due to the fact that the trajectories reaching large distances from the central diffusion region, illustrated in Fig. 2, tend to escape forever. This escape process gives rise, in a time scale that becomes infinitely large upon decrease of the feedback strength, to a diffusion process, namely, to a form of nonequilibrium distribution.

We are convinced that the WS equilibrium is closer to the results of numerical simulation than the Tsallis noncanonical equilibrium. We see, however, that in the case of Fig. 6 the departure of the Tsallis prediction from the results of the numerical simulation is not so marked as to rule out this theoretical proposal. In conclusion, if we use only fitting arguments, we can select the constraint on the second moment rather than that on the first, as the adoption of theoretical arguments would suggest us to do. Eventually, the situation would appear as favorable to Tsallis statistics as illustrated in Fig. 6.

\section{CONCLUDING REMARKS}

The present paper is not just about obtaining the best fit to a numerical simulation. We aim at deriving equilibrium from dynamics with no use of thermodynamic arguments. The result of our investigation is that equilibrium is dictated by the WS statistics rather than by the Tsallis statistics. This yields a satisfactory agreement with the results of numerical simulation with no fitting parameters. The advocates of Tsallis statistics might reach a result as satisfactory as that provided by the dynamic theory (even if we think that actually it is less satisfactory) only by using fitting parameters. This paper results in two important facts. The first is a numerical simulation yielding a noncanonical equilibrium. The second is a theory to account for this noncanonical equilibrium. The theoretical arguments lead us to conclude that the noncanonical equilibrium must correspond to the WS statistics.

If only fitting arguments were to be used, it would be more difficult to make a choice between the Tsallis and the WS statistics. However, if we invoke theoretical arguments also, we find that no room is left for the Tsallis statistics in the case of the dynamic model studied in the present paper. At least within the range of this dynamic model, we show that the noncanonical equilibrium is possible in nature, but it must correspond to the theoretical proposal of West and Seshadri [41] rather than to the predictions of nonextensive thermodynamics [6]. It is interesting to remark that in the limiting case of very weak feedback our model becomes identical to the Lévy flights subject to the Hookean force of the recent work of Jespersen, Metzler, and Fogedby [45], and that both models are equivalent to that originally studied by
West and Seshadri [41]. Also these authors [45] found the Tsallis statistics to be incompatible with the non-Gibbsian nature of the corresponding stationary solution.

In a lucid discussion Lebowitz [30] has recently restated the point of view of Boltzmann, establishing the microscopic origin of irreversible macroscopic behavior. In his view the adoption of the laws of large numbers is essential, and the role of deterministic chaos becomes important only if it applies to a macroscopic number of noninteracting particles. According to Lebowitz, mixing and ergodicity are notions that are "unnecessary, misguided, and misleading." In other words, this opinion reflects the conviction mirrored by the handbooks of statistical mechanics that the unification of mechanics and thermodynamics rests on the condition $N \rightarrow \infty$, where $N$ denotes the number of degrees of freedom of the system under study. These statements do not, however, conflict with the results of Ref. [20] reported in Sec. I B. In fact, the signature of the dynamical origin of thermodynamics, as expressed by Eq. (11), is lost in the limiting case of the thermodynamic limit $N \rightarrow \infty$, thereby making the controversy between the advocates of mixing and the advocates of $N$ $=\infty$ difficult, if not impossible, to substantiate with experimental arguments. This means, in other words, that the canonical equilibrium distribution can be derived using simple arguments based only on probabilistic concepts and the law of large numbers, or if we wish, also on the dynamical arguments of Ref. [20]. If the criterion of simplicity is adopted, one might be tempted to choose the former approach that leads to the wanted result with little or no effort, while the second approach yields the same conclusion after many complicated calculations based on assumed dynamical properties, that only in a few cases has been rigorously proved.

The present paper shows how to extend to the case of boosters with no finite time scale the program of Ref. [20]. In this new case the dynamical approach to equilibrium yields a noncanonical equilibrium, which is that advocated many years ago by West and Seshadri [41]. As pointed out by the theoretical discussion of Sec. III it must be remarked that Eq. (55), yielding the form of equilibrium of West and Seshadri, is the consequence of a form of linear response departing from the traditional wisdom behind the generalized Einstein relation [42]. It is remarkable that the only plausible form of linear response, resting on dynamics, yields the same prescription as that suggested by a phenomenological approach. Thus, while we do agree with Abe and Rajagopal about the fact that the canonical equilibrium is not the only acceptable form of equilibrium, we depart from them on the specific form that this equilibrium will take, since as we have seen in Sec. I A, the Lévy statistics must not be confused with the generalized canonical distribution of Tsallis.
[1] E. W. Montroll and M. F. Shlesinger, in From Stochastics to Hydrodynamics, edited by J. L. Lebowitz and E. W. Montroll, Nonequilibrium Phenomena Vol. II (North-Holland, Amsterdam, 1984).

[2] G. M. Zaslavsky, Phys. Today 52 (8), 39 (1999).
[3] D. H. Zanette and P. A. Alemany, Phys. Rev. Lett. 75, 366 (1995).

[4] C. Tsallis, S. V. F. Levy, A. M. C. Souza, and R. Maynard, Phys. Rev. Lett. 75, 3589 (1995).

[5] M. Buiatti, P. Grigolini, and A. Montagnini, Phys. Rev. Lett. 
82, 3383 (1999).

[6] C. Tsallis, J. Stat. Phys. 52, 479 (1988).

[7] G. Aquino, P. Grigolini, and N. Scafetta, e-print cond-mat/0006245.

[8] P. Grigolini, R. Mannella, and L. Palatella, e-print cond-mat/0011493.

[9] C. Tsallis, R. S. Mendes, and A. R. Plastino, Physica A 261, 534 (1998).

[10] Nonextensive statistical mechanics aims at offering a theoretical framework for systems with long-range interaction, longrange memory, or fractal structure. For its application see, for instance, Br. J. Phys. 29, special issue (1999). A comprehensive list of references is currently available at http:// tsallis.cat.cbpf.br/biblio.htm

[11] D. B. Walton and J. Rafelski, Phys. Rev. Lett. 84, 31 (2000).

[12] G. Wilk and Z. Wlodarczyk, e-print hep-ph/9908459.

[13] S. Abe and A. K. Rajagopal, Phys. Lett. A 272, 341 (2000).

[14] A. Katz, Principles of Statistical Mechanics: the Information Theory Approach (Freeman, San Francisco, 1967).

[15] C. Tsallis, R. S. Mendes, and A. R. Plastino, Physica A 261, 534 (1998).

[16] The rule prescribed by Ref. [15] refers to the case where $x$ is the energy of the system. However, the approach adopted by these authors rest on the information theory [14] and for this reason we can apply it to any "stochastic" variable $x$, whatever is the physical meaning.

[17] The dynamic approach to Lévy statistics [5] is based on the equation of motion $d x / d t=\xi(t)$, where $\xi(t)$ is a stochastic variable with only two values, $W$ and $-W$. The entropic argument is used to derive the waiting-time distribution $\psi(t)$ with a constraint on the first moment of $t>0$. Thus, the function $\Pi(x)$ is related to $\psi(t)$ by the key relation: $\Pi(x)$ $=(1 / 2) \psi(|x| / W) / W$. Therefore, it is now evident that the dynamic approach to Lévy statistics implies a constraint on $|x|$.

[18] E. W. Montroll and B. J. West, in Fluctuation Phenomena, 2nd ed., edited by E. W. Montroll and J. L. Lebowitz, Studies in Statistical Mechanics Vol. 7 (North-Holland, Amsterdam, 1987).

[19] B. V. Gnedenko and A. N. Kolmogorov, Limit Distributions for Sums of Independent Random Variables (Addison-Wesley, Cambridge, 1954).

[20] M. Bianucci, R. Mannella, B. J. West, and P. Grigolini, Phys. Rev. E 51, 3002 (1995).
[21] G. W. Ford, M. Kac, and P. Mazur, J. Math. Phys. 6, 504 (1965).

[22] P. Ullersma, Physica (Utrecht) 32, 27 (1966); 32, 56 (1966); 32, 74 (1966).

[23] P. E. Philipson, J. Math. Phys. 15, 2127 (1987).

[24] D. Vitali and P. Grigolini, Phys. Rev. A 39, 1486 (1989).

[25] K. Lindenberg and B. J. West, The Nonequilibrium Statistical Mechanics of Open and Closed Systems (VCH, New York, 1990).

[26] See Lindenberg and West [25] for a complete review.

[27] K. Huang, Statistical Mechanics (John Wiley, New York, 1987).

[28] E. Fermi, J. Pasta, and S. Ulam, in Collected Works of Enrico Fermi (University of Chicago Press, Chicago, 1965), Vol. II, p. 978.

[29] P. Allegrini, P. Grigolini, and B. J. West, Phys. Rev. E 54, 4760 (1996).

[30] J. L. Lebowitz, Physica A 263, 516 (1999).

[31] D. H. E. Gross and E. Votyakov, e-print cond-mat/9911257.

[32] G. M. Zaslavsky, Physics of Chaos in Hamiltonian Systems on the Foundations of Statistical Physics (Imperial College Press, London, 1998).

[33] T. Geisel and S. Thomae, Phys. Rev. Lett. 52, 1936 (1984).

[34] M. Annunziato, P. Grigolini, and J. Riccardi, Phys. Rev. E 61, 4801 (2000).

[35] M. Annunziato and P. Grigolini, Phys. Lett. A 269, 31 (2000).

[36] M. Bologna, P. Grigolini, and J. Riccardi, Phys. Rev. E 60, 6435 (1999).

[37] P. Gaspard and X.-J. Wang, Proc. Natl. Acad. Sci. U.S.A. 85, 4591 (1988).

[38] M. Ferrario, P. Grigolini, A. Tani, R. Vallauri, and B. Zambon, Adv. Chem. Phys. 62, 225 (1985).

[39] T. Geisel, J. Helstab, and H. Thomas, Z. Phys. B: Condens. Matter 55, 165 (1984).

[40] M. Annunziato, Ph.D. thesis, University of Pisa, 2000.

[41] B. J. West and V. Seshadri, Physica A 113, 203 (1982).

[42] J. P. Bouchaud and A. Georges, Phys. Rep. 195, 127 (1990).

[43] E. Barkai and V. N. Fleurov, Phys. Rev. E 58, 1296 (1998).

[44] G. Trefan, E. Floriani, B. J. West, and P. Grigolini, Phys. Rev. E 50, 2564 (1994).

[45] S. Jespersen, R. Metzler, and H. C. Fogedby, Phys. Rev. E 59, 2736 (1999). 